\title{
Extracción de objeto extraño, por celiotomía en Chelydra serpentina
}

\author{
Karen Vega-Benavides ${ }^{1} \bowtie$, Mauricio Jiménez-Soto ${ }^{1}$, Esteban Rodríguez-Dorado ${ }^{2}$, Emilia Hidalgo- \\ Gómez ${ }^{2}$, Gloria Campos-Alfaro² \\ 1 Hospital Especies Menores y Silvestres, Escuela Medicina Veterinaria, Universidad Nacional. Email: \\ karenluvega@hotmail.com, drjimenezsoto@hotmail.com \\ 2 Estudiante Internado, Hospital Especies Menores y Silvestres, Escuela Medicina Veterinaria, Universidad Nacional. \\ Email: gloriaca1810@gmail.com, emypathi@hotmail.com, rodrguezdorado@gmail.com
}

El impacto antropológico en el ecosistema puede representar un riesgo para los animales de vida libre, no solo respecto a sus nichos, sino también en su alimentación, ya que, al restringirles el acceso a sus dietas naturales, favorecemos la ingesta de dietas dañinas u objetos extraños; además, muchas especies son víctimas del mal manejo que le damos a nuestra basura o desechos, al consumirlos. Los animales con deficiencias nutricionales pueden presentar ingesta incrementada de objetos extraños, por deficiencia de minerales, esto es conocido como "pica". Chelydra serpentina, conocida como tortuga lagarto, es un reptil que se encuentra en áreas con aguas abundantes, con mucha vegetación; es nocturna y se alimenta, principalmente, de peces, anfibios y reptiles menores, moluscos, crustáceos e insectos, además de carroña y ciertos vegetales; su plastrón es pequeño en forma de cruz con puente muy estrecho. La celiotomía, para extracción de cuerpo extraño por gastrostomía, está indicada en caso de cuerpos extraños como anzuelos, ya que la extracción por retracción endoscópica no se recomienda, siendo riesgoso el desgarre del tránsito digestivo comprometido, debe realizarse bajo anestesia general, y con el menor impacto posible en el paciente, preferiblemente no abordar por plastrón, de ser posible. Tortuga, adulta, deprimida y con inapetencia. Radiográficamente se evidencia un anzuelo de pesca en el tracto digestivo, por lo que se decide realizar la extracción quirúrgica, bajo anestesia general. Se realiza pre medicación con midazolam y ketamina, además del manejo analgésico, y mantenimiento con isoflurano (agente inhalatorio). El procedimiento quirúrgico consiste en abordaje de cavidad celómica, se ingresa por el borde lateral izquierdo, craneal al plastrón, sin incidir el mismo; una vez en cavidad se retrae el estómago para ingresar y retirar el anzuelo, el cual se encontraba adherido a pared estomacal, lo cual provoca edema en la zona, posteriormente se cierra la pared estomacal, se realiza lavado en cavidad y se procede al cierre con suturas. En el manejo posquirúrgico se medicó con analgésicos, antibiótico, y se priorizó el cuidado básico de la herida con manejo integral posterior de su habitad y dieta recomendada. La extracción del objeto fue posible por celiotomía lateral, pero se debe considerar que algunos casos deben ser abordados por plastrón, se debe tener en cuenta el tipo de cierre en estos casos. Animales inapetentes, deben ser alimentados por sonda, con dietas indicadas según la especie y condición del paciente; además, el manejo básico de condiciones de habitad, como humedad, luz y temperatura del ambiente. Si bien es cierto, la recuperación pos quirúrgica fue favorable, y un manejo integral ayuda en la recuperación del paciente, este debe continuarse para que la calidad de vida sea la deseable, por lo que el compromiso de sus cuidadores es vital.

凶 Autor para correspondencia Karen Vega-Benavides: karenluvega@hotmail.com 
El aprendizaje en cuanto al manejo médico, quirúrgico, y eco sistémico, de este tipo de paciente, se considera de gran importancia para el personal médico docente y estudiantes de la carrera de veterinaria. Como médicos veterinarios, debemos ser educadores de buenas prácticas de manejo en estos animales, así como orientar a la población a reducir nuestro impacto negativo en las especies silvestres y exóticas. 